\title{
The Impact of Information on Mental Health of Chinese People in the COVID-19
}

\author{
Yangwen Geng, Xiaoli Ni ${ }^{*}$, and Jinming Fan \\ School of Humanities and Social Science, Xi'an Jiaotong University, Xi'an, China
}

\begin{abstract}
As a sudden public event, COVID-19 had caused a great impact on the physical and mental health of people. During this period, people paid great attention to the information of COVID-19. This study investigated the emotional and mental health conditions of 511 residents in the early stage of COVID-19. The results showed that most people were nervous and panic in this period. Individuals who were exposed to more positive information had a lower degree of negative emotional experience; people got more positive and mixed information had significantly better mental health and sleep conditions than people received more negative emotions.
\end{abstract}

\section{Introduction}

\subsection{Mental health in the COVID-19}

The COVID-19 had quickly become an emergency public event in China, due to its diverse transmission routes, long incubation period, strong infectivity, rapid diffusion rate and high mortality rate. While COVID-19 posed a threat to people's lives and mental health [1], people may experience psychological stress reactions such as anxiety, worry and exhaustion. If this stress response was not timely get social support and psychological intervention, it would affect mental health and even turn to psychological stress disorder.Since the outbreak of COVID-19, people all over the world have generally shown a significant increase in depression and anxiety symptoms and pain, and their health conditions have deteriorated [2]. In China, a large-scale national survey, involving 52,739 participants, assessed the psychological burden of the general population during the COVID-19 crisis and found that nearly 35\% of the respondents experienced psychological distress [1]. In addition, while more attention is paid to the psychological problems of infected patients and their families, bereaved persons, and frontline medical staff, the mental health problems of some marginalized people have been largely ignored, such as the elderly and those with chronic diseases, with physical illnesses and with mental illnesses, etc. [3].

* Corresponding author: nixiaolipsy@163.com 


\subsection{Information in the COVID-19}

Social media remains the most indispensable channel of information for most people. During the epidemic, residents increase the frequency and intensity of selecting information and spreading information in social networks. In times of disaster, increased uncertainty may exacerbate people's cognitive dissonance. Therefore, people were more inclined to look for relevant information that can support their emotions and confidence [4]. The media sponsored by the central and local governments at all levels were the most trusted by the public in China, whose rate of being mentioned in the survey was as high as $85.7 \%$ [5]. Authoritative and professional information can correctly guide the public opinion and social mentality, at the same time online rumors, which was fictional and exaggerated epidemic or the shortage of living materials, will aggravate the public panic to different degrees or cause their irrational behavior, and thereby disrupt the normal life of society. Information dissemination had played a role in guiding and regulating public opinion, and the impact on residents should not be underestimated.

\subsection{Information and mental health}

Current research shows that exposure to COVID-19 related information does affect the mental health of residents [6][7]. Studies have shown that crisis communication, refers to "the collection, processing, and dissemination of information required to address a crisis situation”, can convey correct concepts to relieve people's anxiety, so people should pay attention to COVID-19 news reasonably. Reducing exposure to pandemic information may help decline mental health problems related to the COVID-19 [8]. Unfortunately, fake news about COVID-19 spreads as fast as the virus itself. Incorrect health measurements, anxiety and hate speech will have a negative impact on people's physical health and the mental health of the entire world [9]. Overall, during a global pandemic such as COVID-19, effective information delivery can play a key role in reducing fear and uncertainty, while stimulating a unified fight against health threats

At present, many studies have proved that the more information received, the greater the impact on people's mental health, but there is no distinction between the types of information. Therefore, this study intends to study the impact of the positive and negative types of information exposed during the epidemic on residents.

\section{Methods}

\subsection{Participants}

In this study, a questionnaire was issued in February 2020 for an online anonymous survey. A total of 511 valid samples were collected, including 168 male respondents (32.88\% of the total sample) and 343 women (67.12\% of the total sample). Respondents were aged between 13 and 76 years old, with an average age of 33.32 years old.

The occupational composition of the interviewees includes: $42.07 \%$ of full-time students; $28.18 \%$ of professional and technical personnel; $10.37 \%$ of state agencies, enterprises, and business personnel, of which $8.8 \%$ are medical workers. During the epidemic, respondents accounted for $8.8 \%$ of front-line medical workers, $2.2 \%$ of front-line medical staff's families, $2.2 \%$ were required to quarantine at home, $86.5 \%$ of ordinary people, and $3.5 \%$ of other people.

All the data was entered via the Epidata. Statistical analysis adopted the SPSS26.0 statistical analysis software package. 


\subsection{Measures}

\subsubsection{The general information questionnaire}

The general data questionnaire includes demographic information, the type of information, and people's cognitive and emotional conditions about the epidemic. Demographic information includes: gender, age, education level, occupation type, monthly income, marital status, etc.; The type of information was obtained by asking the participants "Which of the following is the type of message you get: (1) There are more positive information; (2) The positive and negativeinformation are almost the same; (3) There are more negative information.”; people's emotional experience assessed with five points, from 1 (no at all) to 5 (very strong), the higher score means the stronger emotional experience.

\subsubsection{Brief Symptom Inventory-18 (BSI-18)}

BSI-18 scale assessed the degree of physical and mental conditions such as depression and anxiety experienced in the past week. The scale contains 18 items, using a five-point Likert-type scale, from 1 (no at all) to 5 (very much), the higher the score, the worse the individual's recent mental health condition. In this sample, internal consistency reliability was good (Cronbach's alpha $=0.938)$.

\subsubsection{Pittsburgh sleep quality index (PSQI)}

There are 8 questions in the scale. The total score of each item is the PSQI score. The score range is $0-24$ points, with $\mathrm{PSQI} \geqslant 8$ points as the standard for judging sleep disorders. The higher the score, the worse the sleep quality. Internal consistency reliability for the present sample was good (Cronbach's alpha $=0.857$ ).

\section{Results}

\subsection{Descriptive statistics}

\subsubsection{Information}

The results showed that half of people (52.3\%) received mixed information (the information they received whether positive or negative are almost the same), $30.1 \%$ of them thought more positive and $17.6 \%$ thought more negative. In terms of sources of information, $72 \%$ of people chose to obtain information from the Internet, who prefer to browse official media, medical professional media and popular self-media platforms. In terms of the frequency of receiving information, $81.6 \%$ said "often" access to browse the COVID-19 information. $15.7 \%$ of them felt the information affected emotions very strongly, $38.7 \%$ thought that it had influence on emotions, $29.7 \%$ held that it had a general effect on emotions; $12.9 \%$ felt that it has little effect , and $2.9 \%$ believed that it was not affect at all.

\subsubsection{Emotional experience}

Most of the 511 subjects expressed nervous and panic in the COVID-19, with only 10.57\% were relatively calm and $5.87 \%$ were very calm; It can be seen from Table 1 that women's 
negative emotion experience was higher than men's, and the difference was statistically significant.

Table 1. Gender differences in emotional experience

\begin{tabular}{cccccc}
\hline \multirow{2}{*}{ Emotional type } & \multicolumn{3}{c}{ Mean } & \multirow{2}{*}{} & $\boldsymbol{p}$ \\
\cline { 2 - 3 } & Men & Women & & $<0.001^{* * *}$ \\
\hline Nervousness & 2.43 & 2.84 & -4.007 & $<0.001^{* * *}$ \\
Fear & 1.99 & 2.63 & -6.257 & $<0.001^{* * *}$ \\
Panic & 1.78 & 2.28 & -5.001 & $0.029^{*}$ \\
Anger & 2.46 & 2.74 & -2.185 & 0.147 \\
Calm & 2.75 & 2.61 & 1.452 & \\
\hline
\end{tabular}

As shown in Table 2, individuals exposed to more negative news experienced higher levels of negative emotions such as anxiety, panic, etc., while individuals got more positive information experienced a lower degree of negative emotion. The four emotions of nervousness, fear, panic, and anger differed to varying degrees due to the types of messages people received, and the differences are statistically significant.

Table 2. Emotional experience with different information types

\begin{tabular}{cccccc}
\hline Emotional type & $\begin{array}{c}\text { More positive } \\
\text { information }\end{array}$ & Mixed information & $\begin{array}{c}\text { More negative } \\
\text { information }\end{array}$ & $\boldsymbol{F}$ & $\boldsymbol{p}$ \\
\hline Nervousness & 2.5 & 2.75 & 2.92 & 4.681 & $0.010^{* *}$ \\
Fear & 2.2 & 2.48 & 2.61 & 4.606 & $0.010^{* *}$ \\
Panic & 1.94 & 2.16 & 2.27 & 3.145 & $0.044^{*}$ \\
Anger & 2.41 & 2.70 & 2.92 & 4.449 & $0.012^{* *}$ \\
Calm down & 2.6 & 2.79 & 2.36 & 1.228 & 0.294 \\
\hline
\end{tabular}

\subsubsection{Sleep quality and mental health conditions}

The positive detective rate of mental health was $17.42 \%$ in this sample, while somatization's positive detective was $4.5 \%$, depression's positive detection rate was $12.9 \%$, anxiety's positive detection rate was $18 \%$, and positive detection rate of mental health in different groups is as shown in Table 3.Compared with the mental health of the ordinary public, the positive detection rate of front-line medical personnel and their families and home quarantine personnel is higher. Among them, the mental health level of home quarantine personnel is the worst. Almost half of home quarantine personnel are in a state of anxiety, and $27 \%$ are in a state of depression.

In the total sample, $62.6 \%$ of them were without sleep disorders, $20.4 \%$ of them were suspected insomnia, and $17 \%$ suffered with insomnia. The detection rates of sleep disorders among people with different groups are shown in Table 3.According to the statistical results, the sleep quality of home quarantine personnel, frontline medical personnel and their family were relatively poor, significantly higher than that of other groups, and there are statistical differences $(\mathrm{F}=3.652, \mathrm{p}<0.05)$.

It can be seen from Table 4 that the BSI-18 scores of people exposed to more negative information were significantly higher than those of the other two groups, and the difference 
was statistically significant $((\mathrm{F}=7.322, \mathrm{p}<0.005)$. Residents exposed to more negative news had significantly higher PSQI scores than the other two groups, and the difference was also statistically significant $((\mathrm{F}=3.225, \mathrm{p}<0.05)$.

Table 3. Detection rates of mental health and sleep disorders in different groups

\begin{tabular}{cccccc}
\hline \multicolumn{2}{c}{ Detection rates } & $\begin{array}{c}\text { Frontline } \\
\text { medical } \\
\text { personnel }\end{array}$ & $\begin{array}{c}\text { Family of the } \\
\text { frontline medical } \\
\text { personnel }\end{array}$ & $\begin{array}{c}\text { Home } \\
\text { quarantine } \\
\text { personnel }\end{array}$ & $\begin{array}{c}\text { Ordinar } \\
\text { y public }\end{array}$ \\
\hline detection rate of & Somatization & $6.70 \%$ & $0 \%$ & $9.10 \%$ & $4.10 \%$ \\
Psychological & Depression & $15.60 \%$ & $18.20 \%$ & $27.30 \%$ & $11.80 \%$ \\
condition & Anxiety & $24.40 \%$ & $18.20 \%$ & $54.50 \%$ & $15.80 \%$ \\
Sleep disorders & No sleep disorder & $48.90 \%$ & $45.50 \%$ & $54.50 \%$ & $64.70 \%$ \\
Detection rate & Suspected insomnia & $22.20 \%$ & $27.30 \%$ & $36.40 \%$ & $20.40 \%$ \\
& Insomnia & $28.90 \%$ & $27.30 \%$ & $9.10 \%$ & $14.90 \%$ \\
\hline
\end{tabular}

Table 4. The mental health and sleep conditions with different types of information

\begin{tabular}{cccccc}
\hline & $\begin{array}{c}\text { More positive } \\
\text { information }\end{array}$ & Mixed information & $\begin{array}{c}\text { More negative } \\
\text { information }\end{array}$ & $\boldsymbol{F}$ & $\boldsymbol{p}$ \\
\hline BSI-18 score & 22.67 & 24.36 & 27.37 & 7.322 & 0.001 \\
PSQI score & 3.13 & 3.49 & 4.36 & 3.225 & 0.041 \\
\hline
\end{tabular}

\section{Discussions}

As a sudden public health event, people had showed high attention and frequency to the information related to the epidemic. Residents had obtained more relevant information from the Internet and pay more attention to the information of official media and medical professional media. It was seen that the effect of centralized communication was immediate and effective. During the investigation, most Chinese were requested to stay at home, which increased residents' attention to the epidemic and frequency of browsing relevant information. The results showed that the different types of information about the epidemic directly affect their emotional experience, mental health, and sleep conditions.

In face of such a public emergency, the main mood of people was nervous and panic, and the negative mood was more serious. At the same time, the positive detection rate of anxiety symptoms was particularly high, and $37.4 \%$ of the people suffered insomnia or suspected insomnia. The results of this study showed that the emotional experience, mental health and sleep conditions of people who are exposed to positive or mixed information are significantly better than those who receive more negative news. Therefore, in such a critical moment, people should pay more attention to positive information, which can appropriately alleviate negative emotional experience. The fact is that public expects and recognizes the authority, credibility, and professionalism of such a communication center in the attention of such an authoritative and professional media platform in a special period. Therefore, media platforms can also spread more timely and correct news to help the public to resolve uncertainty and anxiety.

There are also significant differences in the mental health and sleep conditions of people in different group in the epidemic. In this study, home quarantine personnel had the worst mental health and sleep condition. Since this group was paid great attention, they needed take care of their physical conditions and still facing tremendous psychological pressure 
from themselves and surroundings. Therefore, nearly half of them in this group are anxious, and their sleep status is not optimistic.

Moreover, the mental health status of frontline medical personnel needs more attention. The positive detection rate of the mental health status of this group is generally higher than that of ordinary people, and the sleep status is also the worst among the four groups. Not only they need to rest physically, but also their mental health cannot be ignored after the high-intensity of work.

\section{Limitation and future research}

The type of information is just one of the many factors that affect people's mental health. There are many factors that affect people's emotional experience and mental health, such as the subject's mental state, personality characteristics, previous beliefs, resilience, etc. Therefore, in future research, the influence of these factors should also be considered comprehensively.

\section{References}

1. J. Qiu, B. Shen, M. Zhao, Z. Wang, B. Xie, Y. Xu, Gen. Psychiatry, 33 (2020)

2. Bäuerle, J. Steinbach, A. Schweda, J. Beckord, M. Hetkamp, B. Weismüller, H. Kohler, V. Musche, N. Dörrie, M. Teufel, EM. Skoda, J Prim Care Community Health, 11 (2020)

3. D. Peng, Z. Wang, Y. Xu, Gen. Psychiatry, 33 (2020)

4. L.Peng, Journalism \& Mass Communication Monthly, 5 (2020)

5. N. Wei, C. Yang, X. Wang, Jiangsu Social Sciences, 3 (2020)

6. R.Piltch-Loeb, A. Merdjanoff, G. Meltzer, Prev Med, 145 (2021)

7. P. Jain, J HEALTH COMMUN , 26 (2021)

8. Z. Su, D. McDonnell, J. Wen, M. Kozak, J. Abbas, S. Šegalo, X. Li, J. Ahmad, A. Cheshmehzangi, Y. Cai, L. Yang, Y. Xiang, Global Health, 17 (2021)

9. Y. Li, B. Jiang, K. Shu and H. Liu, in Proceedings of 2020 IEEE International Conference on Big Data (Big Data), 10-13 Dec 2020, taking place virtually(2020) 